\title{
The Dominating Set of Bergman Spaces
}

\author{
Xin Song \\ Hebei University of Technology, Tianjin, China \\ Email: songxin1752@163.com
}

How to cite this paper: Song, X. (2019) The Dominating Set of Bergman Spaces. Journal of Applied Mathematics and Physics, 7, 1560-1571.

https://doi.org/10.4236/jamp.2019.77106

Received: June 18, 2019

Accepted: July 22, 2019

Published: July 25, 2019

Copyright (C) 2019 by author(s) and Scientific Research Publishing Inc. This work is licensed under the Creative Commons Attribution International License (CC BY 4.0).

http://creativecommons.org/licenses/by/4.0/

\begin{abstract}
The dominating set of the weighted Bergman space in the unit disk is characterized in terms of the pseudo-hyperbolic metric disk. Our method is to generalize Luecking's three key lemmas on Bergman space to the weighted Bergman space in the unit disk. We then apply those three lemmas to give a complete description of the dominating set of the weighted Bergman space.
\end{abstract}

\section{Keywords}

Bergman Space, Carleson Measure, Reverse Carleson Inequality

\section{Introduction}

Let $\mathbb{D}$ be the open unit disk in the complex plane $\mathbb{C}$ and let $\mathrm{d} A$ be the Lebesgue area measure on $\mathbb{D}$. For $\alpha>-1$ the weighted Lebesgue measure $\mathrm{d} A_{\alpha}$ is defined by

$$
\mathrm{d} A_{\alpha}=c_{\alpha}\left(1-|z|^{2}\right)^{\alpha} \mathrm{d} A(z),
$$

where

$$
c_{\alpha}=\frac{\Gamma(2+\alpha)}{\Gamma(\alpha+1)}
$$

is a normalizing constant so that

$$
A_{\alpha}(\mathbb{D})=\int_{\mathbb{D}} \mathrm{d} A_{\alpha}=1 .
$$

If $\mu$ is a positive measure on $\mathbb{D}$ and $p>0$, we denote $L^{p}(\mu)$ the Lebesgue space over $\mathbb{D}$ with respect to $\mu$. That is, $L^{p}(\mu)$ consists of all functions $f$ defined on $\mathbb{D}$ for which

$$
\|f\|_{L^{p}(\mu)}=\left(\int_{\mathbb{D}}|f(z)|^{p} \mathrm{~d} \mu(z)\right)^{1 / p}<\infty .
$$

For $\alpha>-1$ and $p>0$, the weighted Bergman space $\mathcal{A}_{\alpha}^{p}(\mathbb{D})$ is defined by 
$\mathcal{A}_{\alpha}^{p}(\mathbb{D})=H(\mathbb{D}) \cap L^{p}\left(\mathbb{D}, \mathrm{d} A_{\alpha}\right)$, where $H(\mathbb{D})$ is the space of all analytic functions on $\mathbb{D}$. That is $f \in \mathcal{A}_{\alpha}^{p}(\mathbb{D})$ if it is holomorphic and

$$
\|f\|_{\mathcal{A}_{\alpha}^{p}(\mathbb{D})}:=\left(\int_{\mathbb{D}}|f(z)|^{p} \mathrm{~d} A_{\alpha}(z)\right)^{\frac{1}{p}}<\infty .
$$

For any $a \in \mathbb{D}$ and $r \in(0,1)$ we write

$$
\Delta(a, r)=\{z \in \mathbb{D}: \rho(z, a)<r\},
$$

where $\rho(z, a)=\left|\varphi_{a}(z)\right|$ is the pseudo-hyperbolic metric and $\varphi_{a}(z)=(a-z) /(1-\bar{a} z)$.

Let $I: \mathcal{A}^{p}(\mathbb{D}) \rightarrow L^{q}(\mathrm{~d} \mu)$ be an identity, we say $\mu$ is a $\mathcal{A}^{p}(\mathbb{D})$-Carleson measure, if there is a constant $C>0$ such that

$$
\|f\|_{\mathcal{A}^{p}(\mathbb{D})} \leq C\|I f\|_{L^{q}(\mathrm{~d} \mu)}
$$

for each $f \in \mathcal{A}^{p}(\mathbb{D})$.

Now we define the dominanting set of Bergman spaces.

Lemma 1 Let $p>0, \alpha>0$ and $G$ be a Lebesgue measurable subset of $\mathbb{D}$. We call $G$ is a dominanting set of $\mathcal{A}_{\alpha}^{p}(\mathbb{D})$ if there is a constant $C>0$ such that

$$
\int_{\mathbb{D}}|f|^{p} \mathrm{~d} A_{\alpha} \leq C \int_{G}|f|^{p} \mathrm{~d} A_{\alpha}
$$

for all $f \in \mathcal{A}_{\alpha}^{p}(\mathbb{D})$.

Let $\chi_{G}$ is the characteristic function of $G$. According to the definition of dominanting set, measure $\mathrm{d} \mu=\chi_{G} \mathrm{~d} v$ satisfies the reverse inequality in Carleson measure definition, that is we have

$$
\int_{\mathbb{D}}|f|^{p} \mathrm{~d} A_{\alpha} \leq C \int_{\mathbb{D}}|f|^{p} \mathrm{~d} \mu
$$

for all $f \in \mathcal{A}_{\alpha}^{p}(\mathbb{D})$. We call reverse Carleson measure if the measure satidfy reverse Carleson inequality. The purpose of this paper is to study reverse $\mathcal{A}_{\alpha}^{p}(\mathbb{D})$ -Carleson measure. [1] and [2] provide some basic tools of Bergman space and some analytic functions. [3] proved Carleson-type embedding theorems for weighted Bergman spaces with Bkoll weights. In 1985, Luecking [4] first studied the reverse Carleson measure in Bergman space on unit disk. The main research tool of Luecking is the dominanting set in Bergman space. In [5], Luecking introduces the necessary and sufficient conditions for dominating sets in Bergman spaces on a unit disk. In recent years, Lou and Zhuo [6] generalized this work to Fock space and gave the characterization of dominant set in Fock space. The purpose of this paper is to extend dominating set and reverse Carleson measure to weighted Bergman spaces. We can find some other concepts in [7] [8] [9] [10] [11]. [12] is a survey on reverse Carleson measures for various Hilbert spaces of analytic functions. We can use some definitions and proof methods in the paper to prove our results. [13] discussed direct and reverse Carleson measures for the de Branges-Rovnyak spaces $\mathcal{H}(b)$. We can refer to their method. In [14], Korhonen and Rättyä has proved the sampling measure by using dominating set and 
$p$-Carleson measure for weighted Bergman space with a weight $\omega$, reference resources [15] [16] [17] [18].

The main results is as follows.

Theorem 2 Suppose $p>0$. Then $G$ is a dominanting set of $\mathcal{A}_{\alpha}^{p}(\mathbb{D})$ if and only if there are constant $\delta>0$ and $0<\eta<1$ such that

$$
A_{\alpha}(G \cap \Delta(a, \eta))>\delta A_{\alpha}(\Delta(a, \eta))
$$

for all set $\Delta(a, \eta)$ and all $a \in \mathbb{D}$.

In Section 2, we mainly give several key lemmas which can prove the main result. In Section 3, we prove the main theorem by using the lemma obtained in Section 2. Section 4 gives the conclusions of this paper and explains how to extend these results to other directions.

\section{Preliminaries}

In this section we collect several technical lemmas that we will need for the proof of our main result. We used the convention that the letter $C$ denotes a constant which may differ from one occurrence to the next.

Lemma 3 (Exercise 1.1.3 (b) in) Let $\mu$ be a Borel measure with $\mu(X)=1$. We have

$$
\left(\int_{X}|f(x)|^{p} \mathrm{~d} \mu(x)\right)^{1 / p} \geq \exp \left(\int_{X} \log |f(x)| \mathrm{d} \mu(x)\right) .
$$

Lemma 4 (Lemma 1.24 in) For any real $\alpha$ and positive $r$ there is constant $C>0$ and $c>0$ such that

$$
c\left(1-|z|^{2}\right)^{2+\alpha} \leq A_{\alpha}(\Delta(z, r)) \leq C\left(1-|z|^{2}\right)^{2+\alpha}
$$

for all $z \in \mathbb{D}$.

Lemma 5 (Lemma 2.20 in) For each $r>0$ there is a positive constant $C_{r}$ such that

$$
C_{r}^{-1} \leq \frac{1-|a|^{2}}{1-|z|^{2}} \leq C_{r}
$$

and

$$
C_{r}^{-1} \leq \frac{1-|a|^{2}}{|1-a \bar{z}|} \leq C_{r}
$$

for all $a$ and $z$ in $\mathbb{D}$ with $\rho(a, z)<r$. Moreover, if $r$ is bounded above, then we may choose $C_{r}$ to be independent of $r$.

Lemma 6 (Corollary 2.21 in) Suppose $-\infty<\alpha<\infty, r_{1}>0, r_{2}>0$ and $r_{3}>0$. Then there is a constant $C>0$ such that

$$
C^{-1} \leq \frac{A_{\alpha}\left(\Delta\left(z, r_{1}\right)\right)}{A_{\alpha}\left(\Delta\left(w, r_{2}\right)\right)} \leq C
$$

for all $z$ and $w$ in $\mathbb{D}$ with $\rho(z, w) \leq r_{3}$.

Lemma 7 (Lemma 2.24 in) Suppose $r>0, p>0$ and $\alpha>-1$. Then there 
is a constant $C>0$ such that

$$
|f(z)|^{p} \leq \frac{C}{\left(1-|z|^{2}\right)^{2+\alpha}} \int_{\Delta(z, r)}|f(w)|^{p} \mathrm{~d} A_{\alpha}(w)
$$

for all $f \in H(\mathbb{D})$ and all $z \in \mathbb{D}$. Moreover we can obtain

$$
|f(z)|^{p} \leq \frac{C_{0}}{A_{\alpha}(\Delta(z, r))} \int_{\Delta(z, r)}|f(w)|^{p} \mathrm{~d} A_{\alpha}(w)
$$

for all $z \in \mathbb{D}$ where $f$ is holomorphic and $C_{0}$ is a constant independent of $f$ and $z$.

If the analytic function $f \in \mathbb{D}$ and $0<\lambda<1$ we consider the local level sets of $f$.

$$
\mathrm{E}_{\lambda}(a)=\mathrm{E}_{\lambda}(f, a)=\{\mathrm{z} \in \Delta(a, \eta):|f(z)|>\lambda|f(a)|\}
$$

and the operator

$$
B_{\lambda} f(a)=\frac{C_{0}}{A_{\alpha}\left(\mathrm{E}_{\lambda}(a)\right)} \int_{\mathrm{E}_{\lambda}(a)}|f|^{p} \mathrm{~d} A_{\alpha}
$$

where $C_{0}$ is in Lemma 7.

By Lemma 7, we can get a inequality

$$
\begin{aligned}
|f(a)|^{p} & \leq C_{0} \frac{\int_{\mathrm{E}_{\lambda}(a)}|f|^{p} \mathrm{~d} A_{\alpha}}{A_{\alpha}(\Delta(a, \eta))} \frac{\int_{\Delta(a, \eta)}|f|^{p} \mathrm{~d} A_{\alpha}}{\int_{\mathrm{E}_{\lambda}(a)}|f|^{p} \mathrm{~d} A_{\alpha}} \\
& =C_{0} \frac{\int_{\mathrm{E}_{\lambda}(a)}|f|^{p} \mathrm{~d} A_{\alpha}}{A_{\alpha}(\Delta(a, \eta))} \frac{\int_{\Delta(a, \eta) \mathrm{E} \mathrm{E}_{\lambda}(a)}|f|^{p} \mathrm{~d} A_{\alpha}+\int_{\mathrm{E}_{\lambda}(a)}|f|^{p} \mathrm{~d} A_{\alpha}}{\int_{\mathrm{E}_{\lambda}(a)}|f|^{p} \mathrm{~d} A_{\alpha}} \\
& \leq C_{0} \frac{\int_{\mathrm{E}_{\lambda}(a)}|f|^{p} \mathrm{~d} A_{\alpha}}{A_{\alpha}(\Delta(a, \eta))}\left(1+\frac{\lambda^{p}|f(a)|^{p} \int_{\Delta(a, \eta) \backslash \mathrm{E}_{\lambda}(a)} \mathrm{d} A_{\alpha}}{\lambda^{p}|f(a)|^{p} \int_{\mathrm{E}_{\lambda}(a)} \mathrm{d} A_{\alpha}}\right) \\
& =C_{0} \frac{\int_{\mathrm{E}_{\lambda}(a)}|f|^{p} \mathrm{~d} A_{\alpha}}{A_{\alpha}\left(\mathrm{E}_{\lambda}(a)\right)}=B_{\lambda} f(a)
\end{aligned}
$$

We can use the same measure as in [5] to prove the following two Lemmas.

Lemma 8 Let $f$ is analytic in $\mathbb{D}$, there is a constant $C_{0}>0$ in Lemma 7 such that

$$
C_{1}=\left\{\begin{array}{lc}
0 & C_{0} \geq 1, \\
\log \frac{1}{C_{0}} & 0<C_{0}<1,
\end{array}\right.
$$

then

$$
\frac{A_{\alpha}\left(\mathrm{E}_{\lambda}(a)\right)}{A_{\alpha}(\Delta(a, \eta))} \geq \frac{\log \frac{1}{\lambda^{p}}+\log \frac{1}{C_{0}}}{\log \frac{B_{\lambda} f(a)}{|f(a)|^{p}}+\log \frac{1}{\lambda^{p}}+C_{1}} .
$$


for all $a \in \mathbb{D}$.

Proof. Applying Lemma 7 and elementary estimates we have

$$
\begin{aligned}
\log |f(a)|^{p} \leq & \log C_{0}+\frac{1}{A_{\alpha}(\Delta(a, \eta))} \int_{\Delta(a, \eta)} \log |f(z)|^{p} \mathrm{~d} A_{\alpha}(z) \\
= & \log C_{0}+\frac{1}{A_{\alpha}(\Delta(a, \eta))}\left[\int_{\Delta(a, \eta) \mathrm{E}_{\lambda}(a)}+\int_{\mathrm{E}_{\lambda}(a)}\right] \log |f(z)|^{p} \mathrm{~d} A_{\alpha}(z) \\
\leq & \log C_{0}+\left[1-\frac{A_{\alpha}\left(\mathrm{E}_{\lambda}(a)\right)}{A_{\alpha}(\Delta(a, \eta))}\right] \log \lambda^{p}|f(a)|^{p} \\
& +\frac{A_{\alpha}\left(\mathrm{E}_{\lambda}(a)\right)}{A_{\alpha}(\Delta(a, \eta))} \frac{1}{A_{\alpha}\left(\mathrm{E}_{\lambda}(a)\right)} \int_{\mathrm{E}_{\lambda}(a)} \log |f(z)|^{p} \mathrm{~d} A_{\alpha}(z) \\
\leq & \log C_{0}+\left[1-\frac{A_{\alpha}\left(\mathrm{E}_{\lambda}(a)\right)}{A_{\alpha}(\Delta(a, \eta))}\right] \log \lambda^{p}|f(a)|^{p} \\
& +\frac{A_{\alpha}\left(\mathrm{E}_{\lambda}(a)\right)}{A_{\alpha}(\Delta(a, \eta))} \log \frac{C_{0}}{A_{\alpha}\left(\mathrm{E}_{\lambda}(a)\right)} \int_{\mathrm{E}_{\lambda}(a)}|f|^{p} \mathrm{~d} A_{\alpha}-\frac{A_{\alpha}\left(\mathrm{E}_{\lambda}(a)\right)}{A_{\alpha}(\Delta(a, \eta))} \log C_{0} \\
\leq & \log C_{0}+\left[1-\frac{A_{\alpha}\left(\mathrm{E}_{\lambda}(a)\right)}{A_{\alpha}(\Delta(a, \eta))}\right] \log \lambda^{p}|f(a)|^{p} \\
& +\frac{A_{\alpha}\left(\mathrm{E}_{\lambda}(a)\right)}{A_{\alpha}(\Delta(a, \eta))} \log B_{\lambda} f(a)-\frac{A_{\alpha}\left(\mathrm{E}_{\lambda}(a)\right)}{A_{\alpha}(\Delta(a, \eta))} \log C_{0}
\end{aligned}
$$

where the last inequality follows by Lemma 3. If we subtract $\log |f(a)|^{p}$ from both sides we get

$$
\begin{aligned}
0 \leq & \log C_{0}+\left[1-\frac{A_{\alpha}\left(\mathrm{E}_{\lambda}(a)\right)}{A_{\alpha}(\Delta(a, \eta))}\right] \log \lambda^{p}|f(a)|^{p}+\frac{A_{\alpha}\left(\mathrm{E}_{\lambda}(a)\right)}{A_{\alpha}(\Delta(a, \eta))} \log B_{\lambda} f(a) \\
& +\frac{A_{\alpha}\left(\mathrm{E}_{\lambda}(a)\right)}{A_{\alpha}(\Delta(a, \eta))} \log \frac{1}{C_{0}}-\log |f(a)|^{p} \\
\leq & \log C_{0}+\left[1-\frac{A_{\alpha}\left(\mathrm{E}_{\lambda}(a)\right)}{A_{\alpha}(\Delta(a, \eta))}\right] \log \lambda^{p}+\frac{A_{\alpha}\left(\mathrm{E}_{\lambda}(a)\right)}{A_{\alpha}(\Delta(a, \eta))} \log \frac{B_{\lambda} f(a)}{|f(a)|^{p}} \\
& +\frac{A_{\alpha}\left(\mathrm{E}_{\lambda}(a)\right)}{A_{\alpha}(\Delta(a, \eta))} \log \frac{1}{C_{0}} .
\end{aligned}
$$

Then we have

$$
-\log C_{0}-\log \lambda^{p} \leq \frac{A_{\alpha}\left(\mathrm{E}_{\lambda}(a)\right)}{A_{\alpha}(\Delta(a, \eta))}\left(\log \frac{1}{\lambda^{p}}+\log \frac{B_{\lambda} f(a)}{|f(a)|^{p}}+\log \frac{1}{C_{0}}\right) .
$$

We notice that $\log \lambda^{p}<0, \log \left(\frac{B_{\lambda} f(a)}{|f(a)|^{p}}\right)>0$ and $\log \frac{1}{C_{0}} \leq C_{1}$. Then we get

$$
\frac{A_{\alpha}\left(\mathrm{E}_{\lambda}(a)\right)}{A_{\alpha}(\Delta(a, \eta))} \geq \frac{\log \frac{1}{\lambda^{p}}+\log \frac{1}{C_{0}}}{\log \frac{B_{\lambda} f(a)}{|f(a)|^{p}}+\log \frac{1}{\lambda^{p}}+C_{1}} .
$$


Lemma 9 Suppose $\alpha>-1$ and $f \in \mathcal{A}_{\alpha}^{p}(\mathbb{D})$. Then let

$$
A=\left\{a \in \mathbb{D}:|f(a)|^{p}<\frac{\varepsilon}{A_{\alpha}(\Delta(a, \eta))} \int_{\Delta(a, \eta)}|f|^{p} \mathrm{~d} A_{\alpha}\right\}
$$

for $\varepsilon>0$ and $\eta \in(0,1)$. There is a constant $C$ depending only on $\eta$, such that

$$
\int_{A}|f|^{p} \mathrm{~d} A_{\alpha} \leq C \varepsilon \int_{\mathbb{D}}|f|^{p} \mathrm{~d} A_{\alpha}
$$

for all $\varepsilon>0$.

Proof. For $a \in A$ we have

$$
|f(a)|^{p} \leq \varepsilon \int_{\mathbb{D}}|f(z)|^{p} \frac{1}{A_{\alpha}(\Delta(a, \eta))} \chi_{\Delta(a, \eta)}(z) \mathrm{d} A_{\alpha}(z) .
$$

Integrate over $a \in A$ and use Fubini's Theorem on the right to obtain

$$
\begin{aligned}
& \int_{A}|f(a)|^{p} \mathrm{~d} A_{\alpha}(a) \\
& \leq \int_{A} \varepsilon \int_{\mathbb{D}}|f(z)|^{p} \frac{1}{A_{\alpha}(\Delta(a, \eta))} \chi_{\Delta(a, \eta)}(z) \mathrm{d} A_{\alpha}(z) \mathrm{d} A_{\alpha}(a) \\
& \leq C \varepsilon \int_{\mathbb{D}}|f(z)|^{p}\left[\int_{A} \frac{1}{A_{\alpha}(\Delta(a, \eta))} \chi_{\Delta(a, \eta)}(z) \mathrm{d} A_{\alpha}(a)\right] \mathrm{d} A_{\alpha}(z) \\
& \leq C \varepsilon \int_{\mathbb{D}}|f(z)|^{p} \mathrm{~d} A_{\alpha}(z)
\end{aligned}
$$

where the second inequality above follows from Lemma 6 and the fact that $\chi_{\Delta(z, r)}(a)=\chi_{\Delta(a, r)}(z)$.

Lemma 10 Let $\varepsilon>0$ and $f \in \mathcal{A}_{\alpha}^{p}(\mathbb{D})$ for $\alpha>-1$. Define the set

$$
B=\left\{a \in \mathbb{D}:|f(a)|^{p}<\varepsilon^{2 / p+1} B_{\lambda} f(a)\right\} .
$$

Then there is a constant $C$ depending only on $\eta$ and $p$, such that

$$
\int_{B}|f|^{p} \mathrm{~d} A_{\alpha} \leq C \varepsilon \int_{\mathbb{D}}|f|^{p} \mathrm{~d} A_{\alpha}
$$

for all $\varepsilon>0$.

Proof. Let $A$ be as in Lemma 9. We write

$$
\int_{B}|f|^{p} \mathrm{~d} A_{\alpha}=\int_{B \cap A}|f|^{p} \mathrm{~d} A_{\alpha}+\int_{B \backslash A}|f|^{p} \mathrm{~d} A_{\alpha} .
$$

The first integral can be estimated by Lemma 9. For the second integral, we have

$$
\begin{aligned}
& \int_{B \backslash A}|f(a)|^{p} \mathrm{~d} A_{\alpha}(a) \leq \int_{B \backslash A} \varepsilon^{2 / p+1} B_{\lambda} f(a) \mathrm{d} A_{\alpha}(a) \\
& =\int_{B \backslash A} \varepsilon^{2 / p+1} \frac{1}{A_{\alpha}\left(\mathrm{E}_{\lambda}(a)\right)} \int_{\mathrm{E}_{\lambda}(a)}|f(z)|^{p} \mathrm{~d} A_{\alpha}(z) \mathrm{d} A_{\alpha}(a) \\
& \leq C \varepsilon^{2 / p+1} \int_{\mathbb{D}}|f(z)|^{p}\left[\int_{B \backslash A} \frac{1}{A_{\alpha}\left(\mathrm{E}_{\lambda}(a)\right)} \chi_{\mathrm{E}_{\lambda}(a)}(z) \mathrm{d} A_{\alpha}(a)\right] \mathrm{d} A_{\alpha}(z) .
\end{aligned}
$$

We need only show the inner integral is suitably bounded. The sets $\mathrm{E}_{\lambda}(a)$ appeared in [5], and Luecking proved in that paper that there is a constant $C>0$, depending only on $p$, such that 


$$
\frac{\left|\mathrm{E}_{\lambda}(a)\right|}{|\Delta(a, r)|} \geq \frac{\epsilon^{2 / p}}{C}
$$

Thus we can obtain

$$
\frac{\left|\mathrm{E}_{\lambda}(a)\right|}{|\Delta(a, r)|}=\frac{\left(1-|a|^{2}\right)^{\alpha}\left|\mathrm{E}_{\lambda}(a)\right|}{\left(1-|a|^{2}\right)^{\alpha}|\Delta(a, r)|} \leq C \frac{\int_{\mathrm{E}_{\lambda}(a)}\left(1-|z|^{2}\right)^{\alpha} \mathrm{d} A(z)}{\int_{\Delta(a, r)}\left(1-|z|^{2}\right)^{\alpha} \mathrm{d} A(z)}=C \frac{A_{\alpha}\left(\mathrm{E}_{\lambda}(a)\right)}{A_{\alpha}(\Delta(a, \eta))}
$$

Combining this with inequality (5), we get

$$
\frac{A_{\alpha}\left(\mathrm{E}_{\lambda}(a)\right)}{A_{\alpha}(\Delta(a, \eta))} \geq \frac{\epsilon^{2 / p}}{C} \text {. }
$$

Plug this into (4) and use $\chi_{\Delta(a, r)}(z)=\chi_{\Delta(z, r)}(a)$. We obtain

$$
\int_{B \backslash A}|f(a)|^{p} \mathrm{~d} A_{\alpha}(a) \leq C \varepsilon \int_{\mathbb{D}}|f(z)|^{p} \mathrm{~d} A_{\alpha}(z) .
$$

\section{Proof of Main Theorem}

We can now characterize a special family of reverse Carleson measures for weighted Bergman spaces with the weighted Lebesgue measure. The main results is as follows.

Theorem 11 Suppose $p>0$. Then $G$ is a dominanting set of $\mathcal{A}_{\alpha}^{p}(\mathbb{D})$ if and only if there are constant $\delta>0$ and $0<\eta<1$ such that

$$
A_{\alpha}(G \cap \Delta(a, \eta))>\delta A_{\alpha}(\Delta(a, \eta))
$$

for all set $\Delta(a, \eta)$ and all $a \in \mathbb{D}$.

Proof. First, we proof the necessity of the Theorem. Take $0<\eta<1$ so that

$$
\int_{|z|<\eta} 1 \mathrm{~d} A_{\alpha}(z)>1-\frac{1}{2 C} \text {. }
$$

By a change of variables, we get

$$
\int_{\Delta(a, \eta)}\left(\frac{1-|a|^{2}}{|1-z \bar{a}|^{2}}\right)^{\alpha+2} \mathrm{~d} A_{\alpha}(z)>1-\frac{1}{2 C} .
$$

Then we can have

$$
\int_{\mathbb{D} \backslash \Delta(a, \eta)}\left(\frac{1-|a|^{2}}{|1-z \bar{a}|^{2}}\right)^{\alpha+2} \mathrm{~d} A_{\alpha}(z)<\frac{1}{2 C} .
$$

Applying (1) to the function

$$
f(z)=\frac{\left(1-|a|^{2}\right)^{(\alpha+2) / p}}{(1-z \bar{a})^{2(\alpha+2) / p}},
$$

we get

$$
\int_{G}\left(\frac{1-|a|^{2}}{|1-z \bar{a}|^{2}}\right)^{\alpha+2} \mathrm{~d} A_{\alpha}(z) \geq \frac{1}{C} \int_{\mathbb{D}}\left(\frac{1-|a|^{2}}{|1-z \bar{a}|^{2}}\right)^{\alpha+2} \mathrm{~d} A_{\alpha}(z)=\frac{1}{C} .
$$


Since $G \cap \Delta(a, \eta)=G-(G \backslash \Delta(a, \eta))$, so we have

$$
\begin{aligned}
& \int_{G \cap \Delta(a, \eta)}\left(\frac{1-|a|^{2}}{|1-z \bar{a}|^{2}}\right)^{\alpha+2} \mathrm{~d} A_{\alpha}(z) \\
& =\int_{G}\left(\frac{1-|a|^{2}}{|1-z \bar{a}|^{2}}\right)^{\alpha+2} \mathrm{~d} A_{\alpha}(z)-\int_{G \backslash \Delta(a, \eta)}\left(\frac{1-|a|^{2}}{|1-z \bar{a}|^{2}}\right)^{\alpha+2} \mathrm{~d} A_{\alpha}(z) \\
& \geq \int_{G}\left(\frac{1-|a|^{2}}{|1-z \bar{a}|^{2}}\right)^{\alpha+2} \mathrm{~d} A_{\alpha}(z)-\int_{\mathbb{D} \backslash \Delta(a, \eta)}\left(\frac{1-|a|^{2}}{|1-z \bar{a}|^{2}}\right)^{\alpha+2} \mathrm{~d} A_{\alpha}(z) \\
& \geq \frac{1}{C}-\frac{1}{2 C}=\frac{1}{2 C} .
\end{aligned}
$$

It is easy to verify that

$$
\frac{\left(1-|a|^{2}\right)^{\alpha+2}}{|1-z \bar{a}|^{2(\alpha+2)}} \leq \frac{\left(1-|a|^{2}\right)^{\alpha+2}}{(1-|a|)^{2(\alpha+2)}} \leq \frac{2^{\alpha+2}}{(1-|a|)^{\alpha+2}} .
$$

Combining this with the above inequality, we get

$$
\frac{2^{\alpha+2}}{(1-|a|)^{\alpha+2}} A_{\alpha}(G \cap \Delta(a, \eta)) \geq \int_{G \cap \Delta(a, \eta)} \frac{\left(1-|a|^{2}\right)^{\alpha+2}}{|1-z \bar{a}|^{2(\alpha+2)}} \mathrm{d} A_{\alpha}(z) \geq \frac{1}{2 C},
$$

so inequality

$$
A_{\alpha}(G \cap \Delta(a, \eta)) \geq \frac{1}{2^{\alpha+3} C}(1-|a|)^{\alpha+2}
$$

which gives (6).

For sufficiency of the theorem,we will follow the arguments in [5] closely. For $\varepsilon>0$ let

$$
F=\mathbb{D} \backslash B=\left\{a \in \mathbb{D}:|f(a)|^{p} \geq \varepsilon^{2 / p+1} B_{\lambda} f(a)\right\} .
$$

According to Lemma 10, we have

$$
\int_{\mathbb{D}}|f|^{p} \mathrm{~d} A_{\alpha}=\left(\int_{F}+\int_{B}\right)|f|^{p} \mathrm{~d} A_{\alpha} \leq \int_{F}|f|^{p} \mathrm{~d} A_{\alpha}+C \varepsilon \int_{\mathbb{D}}|f|^{p} \mathrm{~d} A_{\alpha}
$$

If we now choose $\varepsilon$ small enough so that $\varepsilon C<1 / 2$, we have

$$
\int_{\mathbb{D}}|f|^{p} \mathrm{~d} A_{\alpha}<2 \int_{F}|f|^{p} \mathrm{~d} A_{\alpha} .
$$

By the definition of $F$ we have $B_{\lambda} f(a) /|f(a)|^{p} \leq \varepsilon^{-(2 / p+1)}$ for all $a \in F$.

If $C_{0} \geq 1$, then $C_{1}=0$. Lemma 8 can be write

$$
\frac{A_{\alpha}\left(\mathrm{E}_{\lambda}(a)\right)}{A_{\alpha}(\Delta(a, \eta))} \geq \frac{\log \frac{1}{\lambda^{p}}+\log \frac{1}{C_{0}}}{\log \frac{B_{\lambda} f(a)}{|f(a)|^{p}}+\log \frac{1}{\lambda^{p}}} .
$$

For the $\delta>0$ in (6), we choose a positive integer $k>4 / \delta$, which implies that 


$$
\frac{k+1}{2 k} \delta-\frac{1}{k}>\frac{1}{2} \delta-\frac{1}{4} \delta=\frac{1}{4} \delta .
$$

Therefore, by choosing

$$
\lambda^{p}<\min \left\{\varepsilon^{2(2 / p+1) / \delta}, C_{0}^{-k}\right\}
$$

Lemma 8 gives

$$
\begin{aligned}
\frac{A_{\alpha}\left(\mathrm{E}_{\lambda}(a)\right)}{A_{\alpha}(\Delta(a, \eta))} & \geq \frac{\log \frac{1}{\lambda^{p}}+\log \frac{1}{C_{0}}}{\log \frac{B_{\lambda} f(a)}{|f(a)|^{p}}+\log \frac{1}{\lambda^{p}}} \geq \frac{\frac{k-1}{k} \log \frac{1}{\lambda^{p}}}{\log \frac{B_{\lambda} f(a)}{|f(a)|^{p}}+\log \frac{1}{\lambda^{p}}} \\
& \geq \frac{k-1}{k} \frac{(2 / \delta) \log \frac{1}{\varepsilon^{2 / p+1}}}{\log \frac{1}{\varepsilon^{2 / p+1}}+(2 / \delta) \log \frac{1}{\varepsilon^{2 / p+1}}} \\
& =\frac{k-1}{k} \cdot \frac{2}{2+\delta} \geq \frac{k-1}{k}\left(1-\frac{\delta}{2}\right) .
\end{aligned}
$$

So we have

$$
\begin{aligned}
& A_{\alpha}\left(\mathrm{E}_{\lambda}(a)\right) \geq \frac{k-1}{k}\left(1-\frac{\delta}{2}\right) A_{\alpha}(\Delta(a, \eta)) \\
& =A_{\alpha}(\Delta(a, \eta))-\frac{1}{k}\left(1+\frac{k-1}{2} \delta\right) A_{\alpha}(\Delta(a, \eta)) \\
& =A_{\alpha}\left(\Delta(a, \eta) \backslash \mathrm{E}_{\lambda}(a)\right)+A_{\alpha}\left(\mathrm{E}_{\lambda}(a)\right)-\frac{1}{k}\left(1+\frac{k-1}{2} \delta\right) A_{\alpha}(\Delta(a, \eta)),
\end{aligned}
$$

It implies that

$$
A_{\alpha}\left(\Delta(a, \eta) \backslash \mathrm{E}_{\lambda}(a)\right) \leq \frac{1}{k}\left(1+\frac{k-1}{2} \delta\right) A_{\alpha}(\Delta(a, \eta)) .
$$

Note that

$$
G \cap \mathrm{E}_{\lambda}(a)=G \cap\left(\Delta(a, \eta)-\left[\Delta(a, \eta) \backslash \mathrm{E}_{\lambda}(a)\right]\right),
$$

then following from (6) we have

$$
\begin{aligned}
& A_{\alpha}\left(G \cap \mathrm{E}_{\lambda}(a)\right) \\
& =A_{\alpha}(G \cap \Delta(a, \eta))-A_{\alpha}\left(G \cap\left(\Delta(a, \eta) \backslash \mathrm{E}_{\lambda}(a)\right)\right) \\
& \geq \delta A_{\alpha}(\Delta(a, \eta))-A_{\alpha}\left(\Delta(a, \eta) \backslash \mathrm{E}_{\lambda}(a)\right) \\
& >\delta A_{\alpha}(\Delta(a, \eta))-\frac{1}{k}\left(1+\frac{k-1}{2} \delta\right) A_{\alpha}(\Delta(a, \eta)) \\
& =\left[\frac{k+1}{2 k} \delta-\frac{1}{k}\right] A_{\alpha}(\Delta(a, \eta))>\frac{\delta}{4} A_{\alpha}(\Delta(a, \eta))
\end{aligned}
$$

whenever $a \in F$.

If $0<C_{0}<1$ so taht $C_{1}=\log \frac{1}{C_{0}}$, then $\log \frac{1}{C_{0}}>0$. Lemma 8 can be write 


$$
\frac{A_{\alpha}\left(\mathrm{E}_{\lambda}(a)\right)}{A_{\alpha}(\Delta(a, \eta))} \geq \frac{\log \frac{1}{\lambda^{p}}+\log \frac{1}{C_{0}}}{\log \frac{B_{\lambda} f(a)}{|f(a)|^{p}}+\log \frac{1}{\lambda^{p}}+\log \frac{1}{C_{0}}} \geq \frac{\log \frac{1}{\lambda^{p}}}{\log \frac{B_{\lambda} f(a)}{|f(a)|^{p}}+\log \frac{1}{\lambda^{p}}} .
$$

Therefore, by choosing

$$
\lambda^{p}<\varepsilon^{2(2 / p+1) / \delta},
$$

Lemma 8 gives

$$
\frac{A_{\alpha}\left(\mathrm{E}_{\lambda}(a)\right)}{A_{\alpha}(\Delta(a, \eta))} \geq \frac{\log \frac{1}{\lambda^{p}}}{\log \frac{B_{\lambda} f(a)}{|f(a)|^{p}}+\log \frac{1}{\lambda^{p}}} \geq \frac{2}{2+\delta} \geq 1-\frac{\delta}{2} .
$$

So we have

$$
\begin{aligned}
& A_{\alpha}\left(\mathrm{E}_{\lambda}(a)\right) \geq\left(1-\frac{\delta}{2}\right) A_{\alpha}(\Delta(a, \eta)) \\
& =A_{\alpha}(\Delta(a, \eta))-\frac{\delta}{2} A_{\alpha}(\Delta(a, \eta)) \\
& =A_{\alpha}\left(\Delta(a, \eta) \backslash \mathrm{E}_{\lambda}(a)\right)+A_{\alpha}\left(\mathrm{E}_{\lambda}(a)\right)-\frac{\delta}{2} A_{\alpha}(\Delta(a, \eta))
\end{aligned}
$$

It implies that

$$
A_{\alpha}\left(\Delta(a, \eta) \backslash \mathrm{E}_{\lambda}(a)\right) \leq \frac{\delta}{2} A_{\alpha}(\Delta(a, \eta)) .
$$

Note that

$$
G \cap \mathrm{E}_{\lambda}(a)=G \cap\left(\Delta(a, \eta)-\left[\Delta(a, \eta) \backslash \mathrm{E}_{\lambda}(a)\right]\right),
$$

then following from (6) and (8) we have

$$
\begin{aligned}
& A_{\alpha}\left(G \cap \mathrm{E}_{\lambda}(a)\right) \\
& =A_{\alpha}(G \cap \Delta(a, \eta))-A_{\alpha}\left(G \cap\left(\Delta(a, \eta) \backslash \mathrm{E}_{\lambda}(a)\right)\right) \\
& \geq \delta A_{\alpha}(\Delta(a, \eta))-A_{\alpha}\left(\Delta(a, \eta) \backslash \mathrm{E}_{\lambda}(a)\right) \\
& >\delta A_{\alpha}(\Delta(a, \eta))-\frac{\delta}{2} A_{\alpha}(\Delta(a, \eta)) \\
& =\frac{\delta}{2} A_{\alpha}(\Delta(a, \eta))>\frac{\delta}{4} A_{\alpha}(\Delta(a, \eta))
\end{aligned}
$$

whenever $a \in F$.

Then we can get

$$
A_{\alpha}\left(G \cap \mathrm{E}_{\lambda}(a)\right) \geq \frac{\delta}{4} A_{\alpha}(\Delta(a, \eta))
$$

for all constant $C_{0}>0$ in Lemma 7 .

Hence we have

$$
\begin{aligned}
& \frac{1}{A_{\alpha}(\Delta(a, \eta))} \int_{G} \chi_{\Delta(a, \eta)}(z)|f(z)|^{p} \mathrm{~d} A_{\alpha}(z) \\
& \geq \frac{1}{A_{\alpha}(\Delta(a, \eta))} \int_{G} \chi_{\mathrm{E}_{\lambda}(a)}(z)|f(z)|^{p} \mathrm{~d} A_{\alpha}(z)
\end{aligned}
$$




$$
\begin{aligned}
& \geq \frac{1}{A_{\alpha}(\Delta(a, \eta))} A_{\alpha}\left(G \cap \mathrm{E}_{\lambda}(a)\right) \lambda^{p}|f(a)|^{p} \\
& \geq \frac{1}{4} \delta \lambda^{p}|f(a)|^{p}
\end{aligned}
$$

where $a \in F$. Integrating both side over $F$ and using Fubini's Theorem, we obtain

$$
\int_{G}|f(z)|^{p}\left(\int_{F} \frac{1}{A_{\alpha}(\Delta(a, \eta))} \chi_{\Delta(a, \eta)}(z) \mathrm{d} A_{\alpha}(a)\right) \mathrm{d} A_{\alpha}(z) \geq \frac{1}{4} \delta \lambda^{p} \int_{F}|f|^{p} \mathrm{~d} A_{\alpha} .
$$

The integral in the brackets of the left-hand side can be estimated as follows:

$$
\int_{F} \frac{1}{A_{\alpha}(\Delta(a, \eta))} \chi_{\Delta(a, \eta)}(z) \mathrm{d} A_{\alpha}(a) \leq C \frac{\int_{F \cap \Delta(z, \eta)} \mathrm{d} A_{\alpha}(a)}{A_{\alpha}(\Delta(a, \eta))} \leq C \frac{A_{\alpha}(\Delta(a, \eta))}{A_{\alpha}(\Delta(a, \eta))}=C .
$$

And the right hand side of (9) can be estimated from below using (7). This yields

$$
C \int_{G}|f(z)|^{p} \mathrm{~d} A_{\alpha}(z) \geq \frac{1}{8} \delta \lambda^{p} \int_{\mathbb{D}}|f|^{p} \mathrm{~d} A_{\alpha}
$$

which proves the sufficiency of the theorem.

\section{Conclusions}

We proved the dominating set by using pseudo-hyperbolic metric disk and sub-mean inequality. The method of proof is to obtain the complete characterization of dominating set by applying the key lemma given in Section 2 in Section 3.

Next we will study some applications of Theorem 11. Let $\phi$ be a bounded measurable function on $\mathbb{D}$. So we want to prove that the Toeplitz operator $T_{\phi}$ is bounded. Using dominating set and Carleson measures, we can also study sampling measures for weighted Bergman space.

\section{Conflicts of Interest}

The author declares no conflicts of interest regarding the publication of this paper.

\section{References}

[1] Grafakos, L. (2014) Classical Fourier Analysis. Graduate Texts in Mathematics, Vol. 249, Springer-Verlag, New York. https://doi.org/10.1007/978-1-4939-1194-3

[2] Zhu, K. (2005) Spaces of Holomorphic Functions in the Unit Ball. Graduate Texts in Mathematics, Vol. 226, Springer-Verlag, New York.

[3] Constantin, O. (2010) Carleson Embeddings and Some Classes of Operators on Weighted Bergman Spaces. Journal of Mathematical Analysis and Applications, 365, 668-682. https://doi.org/10.1016/j.jmaa.2009.11.035

[4] Luecking, D. (1985) Forward and Reverse Carleson Inequalities for Functions in Bergman Spaces and Their Derivatives. American Journal of Mathematics, 107, 85-111. https://doi.org/10.2307/2374458

[5] Luecking, D. (1981) Inequalities on Bergman Spaces. Illinois Journal of Mathemat- 
ics, 25, 1-11. https://doi.org/10.1215/ijm/1256047358

[6] Lou, Z. and Zhuo, Z. (2018) A Class of Reverse Carleson Measures on Doubling Fock Spaces. Complex Analysis and Operator Theory, 13, 1795-1809.

https://doi.org/10.1007/s11785-018-0858-6

[7] Garnett, J. (2010) Bounded Analytic Functions. Graduate Texts in Mathematics, Vol. 236, Springer-Verlag, New York.

[8] Hastings, W.W. (1975) A Carleson Measure Theorem for Bergman Spaces. Proceedings of the American Mathematical Society, 52, 237-241.

https://doi.org/10.2307/2040137

[9] Mcdonald, G. and Sundberg, C. (1979) Toeplitz Operators on the Disc. Indiana University Mathematics Journal, 28, 595-611. https://doi.org/10.1512/iumj.1979.28.28042

[10] Hu, Z., Lv, X. and Schuster, A.P. (2019) Bergman Spaces with Exponential Weights. Journal of Functional Analysis, 276, 1402-1429. https://doi.org/10.1016/j.jfa.2018.05.001

[11] Kwon, E.G. (2018) On Carlesons Inequality. Journal of Inequalities and Applications, 91, 1-6. https://doi.org/10.1186/s13660-018-1682-2

[12] Fricain, E., Hartmann, A. and Ross, W.T. (2016) A Survey on Reverse Carleson Measures. Theta Series in Advanced Mathematics, 91-123.

[13] Blandignères, A., Fricain, E., Gaunard, F., Hartmann, A. and Ross, W.T. (2015) Direct and Reverse Carleson Measures for $\mathcal{H}(b)$ Spaces. Indiana University Mathematics Journal, 64, 1027-1057. https://doi.org/10.1512/iumj.2015.64.5604

[14] Korhonen, T. and Rättyä, J. (2019) Zero Sequences, Factorization and Sampling Measures for Weighted Bergman Spaces. Mathematische Zeitschrift, 291, 1145-1173. https://doi.org/10.1007/s00209-019-02243-7

[15] Peláez, J.Á. and Rättyä, J. (2014) Weighted Bergman Spaces Induced by Rapidly Increasing Weights. Memoirs of the American Mathematical Society Book 227. American Mathematical Society, Providence. https://doi.org/10.1016/j.aim.2013.03.006

[16] Peláez, J.Á. and Rättyä, J. (2015) Embedding Theorems for Bergman Spaces via Harmonic Analysis. Mathematische Annalen, 362, 205-239. https://doi.org/10.1007/s00208-014-1108-5

[17] Peláez, J.Á., Rättyä, J. and Sierra, K. (2015) Embedding Bergman Spaces into Tent Spaces. Mathematische Zeitschrift, 281, 1215-1237. https://doi.org/10.1007/s00209-015-1528-2

[18] Peláez, J.Á. (2016) Small Weighted Bergman Spaces. Proceedings of the Summer School in Complex and Harmonic Analysis, and Related Topics, Kuopio, 2014, 29-98. 